\title{
Commentary: Mesothelioma: The Guinness world record holder in thromboembolism
}

\author{
Robert B. Cameron, MD
}

The diagnosis of cancer increases the risk of thromboembolic events (TEEs) by 10 times from the baseline of $0.1 \%$ to $2 \%$ in the general population to $10 \%$ to $15 \%$. ${ }^{1}$ The diagnosis of malignant mesothelioma further increases the risk of TEE by nearly 2 times to as high as $27.7 \%$, with both venous and arterial life-threatening events occurring, such as pulmonary embolism and myocardial infarction. ${ }^{1,2}$ Unrecognized TEE may be responsible for many of the nonperioperative deaths in the early postoperative period (ie, from 30-120 postoperative days). Prevention protocols and quality metrics include perioperative measures, such as sequential compression devices and postoperative medications like heparin administered subcutaneously every 8 to 12 hours. Yet, standard prophylaxis and treatment of clinically evident disease is clearly not enough in these high-risk patients.

The recent study by DeLeon and colleagues ${ }^{3}$ confirms this staggeringly high incidence of TEE in patients with malignant pleural mesothelioma undergoing surgery. In their study, the rate of TEE was determined by a "surveillance" program of weekly postoperative venous duplex studies (VDS) beginning on postoperative day 7 (POD7). Once identified, TEE were treated by anticoagulation for 90 days. The overall incidence of TEE amazingly was found to be $32.2 \%$ (30/93 patients). This included both upper- and lower-extremity venous thromboses as determined by symptoms and VDS and soberingly occurred despite routine TEE prophylactic measures.

\footnotetext{
From the Division of Thoracic Surgery, Department of Surgery, David Geffen School of Medicine at UCLA and the Division of Thoracic Surgery, Department of Surgery and Perioperative Care, West Los Angeles VA Medical Center, Los Angeles, Calif.

Disclosures: Author has nothing to disclose with regard to commercial support.

Received for publication Dec 22, 2019; accepted for publication Dec 22, 2019; available ahead of print Jan 11, 2020.

Address for reprints: Robert B. Cameron, MD, Division of Thoracic Surgery, Department of Surgery, David Geffen School of Medicine, 10780 Santa Monica Blvd, Suite 100, Los Angeles, CA 90025 (E-mail: rcameron@stanfordalumni.org).

J Thorac Cardiovasc Surg 2020;160:1074-5

$0022-5223 / \$ 36.00$

Copyright (c) 2020 by The American Association for Thoracic Surgery

https://doi.org/10.1016/j.jtcvs.2019.12.082
}

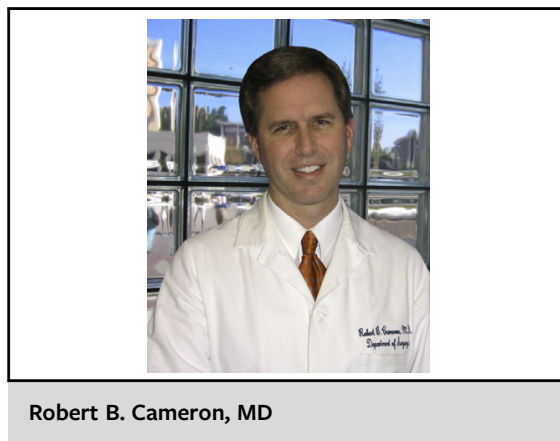

CENTRAL MESSAGE

The risk of thromboembolic

events in patients with meso-

thelioma undergoing surgery is

staggering; more attention needs

to be paid to early detection and

treatment but most importantly

to prevention.

Faced with a problem of this magnitude despite current standard prophylactic measures, mesothelioma surgeons must adopt much more aggressive early detection (and treatment) programs. We cannot be satisfied with simply treating symptomatic patients or patients found to have TEE 7 days following surgery. For decades, surgeons have appreciated that venous thrombosis most often occurs intraoperatively or in the immediate postoperative period. In the "surveillance" protocol described by DeLeon and colleagues, nearly one third (9/30) of TEEs were symptomatic before the first surveillance scan and an additional $56.7 \%$ (17/30) were detected by the first surveillance scan on POD7. Thus, $86.7 \%$ of the TEE (26/30) developed before POD7. Clearly, we must detect these events earlier whenever they occur, perhaps as early as the first 2 to 5 postoperative days. Screening VDS could easily be justified within the first 3 to 5 postoperative days. Although treatment of early postoperative TEE may be more complex with the presence of epidural catheters and a large raw surface area at risk of hemorrhage, aggressive therapy certainly is warranted.

Finally, it is imperative that more effective prophylactic measures are studied and instituted. This may include combinations of sequential compression devices, early mobilization, aspirin, and enoxaparin. Earlier and greater levels of anticoagulation may be required and could force the adoption of alterative pain-management strategies, such as liposomal bupivacaine, paravertebral, and serratus blocks, to avoid the 
limitations associated with epidural catheters. Prophylactic therapy even may need to be extended in all patients from the typical initial inpatient period to a full 90 postoperative days, for instance, with enoxaparin or apixaban. The knowledge that mesothelioma holds the Guinness record for TEE only means that we must be Olympian in our efforts to treat and prevent this complication.

\section{References}

1. Nguyen D, Lee SJ, Libby E, Verschraegen C. Rate of thromboembolic events in mesothelioma. Ann Thorac Surg. 2008;85:1032-8.

2. Koksal D, Safak O, Ozcan A, Agackiran Y, Erturk H, Findik G, et al. Thromboembolic events in malignant pleural mesothelioma. Clin Appl Thromb Hemost. 2016;22:390-4.

3. DeLeon LE, Bravo-Iñiguez CE, Sam Fox S, Tarascio J, Freyaldenhoven S, Lapidot M, et al. Routine surveillance for diagnosis of venous thromboembolism after pleurectomy for malignant pleural mesothelioma. J Thorac Cardiovasc Surg. 2020;160:1064-73.
See Article page 1064.

\section{Commentary: Focused attention on proactive identification of deep venous thrombosis after pleurectomy/decortication for malignant pleural mesothelioma}

\author{
Shawn S. Groth, MD, MS, FACS, \\ R. Taylor Ripley, MD, Philip W. Carrott, MD, and \\ Bryan M. Burt, MD
}

Cytoreductive surgery, either pleurectomy/decortication (PD) or extrapleural pneumonectomy (EPP), plays a central role in the multimodal treatment of malignant pleural mesothelioma. Importantly, the risk of postoperative complications after PD and EPP is significant-up to $60 \%$ after EPP. ${ }^{1}$ In particular, deep venous thrombosis (DVT; incidence, 1.5\%-6.4\%) and pulmonary embolus (PE; incidence, $0.5 \%-4 \%$ ) are especially important. ${ }^{2}$ Early recognition is critical, given the high mortality rate of these complications. In 2 large series of EPPs, including one from Brigham and Women's Hospital, ${ }^{1} \mathrm{PE}$ was the most common cause of 30-day mortality, accounting for $30 \%$ of all deaths. ${ }^{1,3}$ Given this clinical significance, De Leon and colleagues ${ }^{4}$ report a DVT surveillance strategy with the aim of

\footnotetext{
From the Division of Thoracic Surgery, Michael E. DeBakey Department of Surgery, Baylor College of Medicine, Houston, Tex.

Disclosures: Authors have nothing to disclose with regard to commercial support.

Received for publication Dec 20, 2019; accepted for publication Dec 24, 2019; available ahead of print Jan 11, 2020.

Address for reprints: Shawn S. Groth, MD, MS, FACS, Division of Thoracic Surgery, Michael E. DeBakey Department of Surgery, Baylor College of Medicine, One Baylor Plaza, BCM 390, Houston, TX 77030 (E-mail: Shawn.Groth@bcm.edu). J Thorac Cardiovasc Surg 2020;160:1075-6 $0022-5223 / \$ 36.00$

Copyright (c) 2020 by The American Association for Thoracic Surgery https://doi.org/10.1016/j.jtcvs.2019.12.088
}

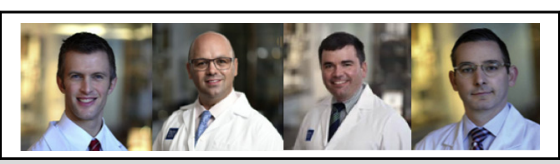

Shawn S. Groth, MD, MS, FACS, R. Taylor Ripley, MD, Philip W. Carrott, MD, and Bryan M. Burt, MD

\section{CENTRAL MESSAGE \\ The patient's outcome corre- lates directly with the surgeon's attention to a myriad of minor details.}

detecting DVTs and PEs early, when the probability of mitigating their negative impact on patient outcomes is greatest. In this edition of the Journal, a prospective evaluation of their surveillance program is presented.

All patients had lower-extremity noninvasive studies at baseline preoperatively and every 7 days postoperatively (or earlier if there were signs of DVT or PE). After excluding 7 patients who were found to have a DVT on their preoperative lower-extremity noninvasive studies, the authors included 93 patients who underwent PD over a 27 -month period in their final analysis. Despite using 5000 unit of subcutaneous heparin every 8 hours for prophylaxis, the authors found a $29 \%$ incidence of postoperative DVT (median time to event, 7 days; range, 1-14 days) and an $11 \%$ incidence of PE (median time to event, 7 days; range 1-23 days). 